\title{
The Industry Research Status of Millimeter-wave 5G
}

\author{
Guoqing $\mathrm{Hu}^{1,2, a,{ }^{*}}$, Jin $\mathrm{He}^{1,2, b}$, Chunlai $\mathrm{Li}^{1}$, Jingjing Liư ${ }^{1}$, Jun Pan ${ }^{1}$, Huali You ${ }^{1}$, \\ Zhijiong Wang ${ }^{1}$ and Ying $\mathrm{Yu}^{1}$ \\ ${ }^{1}$ Peking University Shenzhen SoC Key Laboratory, Peking University Shenzhen Institution, \\ Shenzhen, China \\ 2 School of Electronics Engineering and Computer Science, Peking University, Beijing, China \\ ahuking@pku.edu.cn, bfrankhe@pku.edu.cn \\ *Corresponding author: huking@pku.edu.cn
}

Keywords: Millimeter-wave, Mobile Communications, 5G.

\begin{abstract}
The demand of mass data services brought by the mobile Internet and the Internet of things makes the traditional mobile communication spectrum resources become saturated. The high frequency millimeter-wave $5 \mathrm{G}$ is becoming the subject of extensive research in the industry. This paper selects the most representative five industry companies in China, Europe, the United States , Japan and Korea, the five largest 5G driving countries, and discusses the latest research progress in the field of millimeter-wave 5G. Through analysis, we find that the future $5 \mathrm{G}$ network will use the low frequency $(<6 \mathrm{GHz})$ for wide area coverage and high frequency $(>6 \mathrm{GHz})$ millimeter-wave for hot spot access.
\end{abstract}

\section{毫米波5G产业界研究现状}

胡国庆 ${ }^{1,2, a,}{ }^{*}$, 何进 ${ }^{1,2, b}$, 李春来 ${ }^{1}$, 刘京京 ${ }^{1}$, 潘俊 $^{1}$, 尤华丽 ${ }^{1}$, 王志畑 ${ }^{1}$ ，余迎 ${ }^{1}$ 1 北京大学深圳研究院系统芯片设计重点实验室, 深圳, 中国

2北京大学信息科学技术学院, 北京, 中国

ahuking@pku.edu.cn, bfrankhe@pku.edu.cn

*通讯作者: huking@pku.edu.cn

关键词: 毫米波; 移动通信; 5G。

中文摘要. 移动互联网和物联网带来的庞大数据业务需求, 使得传统移动通信频谱资源趋于 饱和, 高频段毫米波 $5 \mathrm{G}$ 正成为产业界广泛研究的课题。本文选取了中欧美日韩五大 $5 \mathrm{G}$ 推动国 最具代表性的五家产业界公司, 探讨了毫米波 $5 \mathrm{G}$ 领域的最新研究进展。通过分析, 我们发现 未来5G网络将采用低频段（<6 GHz）蜂窝进行广域覆盖、高频段（>6GHz）毫米波进行热点 区域接入相结合的方式。

1. 引言

第一代到第四代移动通信主要提供面向个人应用的移动业务, 而 $5 \mathrm{G}$ 将渗透到未来社会的 各个领域，提供面向产业应用的数据业务，建立完善的信息生态系统。5G将为个人及产业用 户提供高达几十Gbps的峰值接入速率、毫米量级的超低时延用户体验、千亿量级的接入容量, 移动性可支持到 $500 \mathrm{~km} / \mathrm{h}$, 可以为超高流量密度、超密集连接数密度等不同场景提供极致业 务体验，真正做到 “信息随心至、万物触手及”。5G的总体业务愿景如图1所示。 


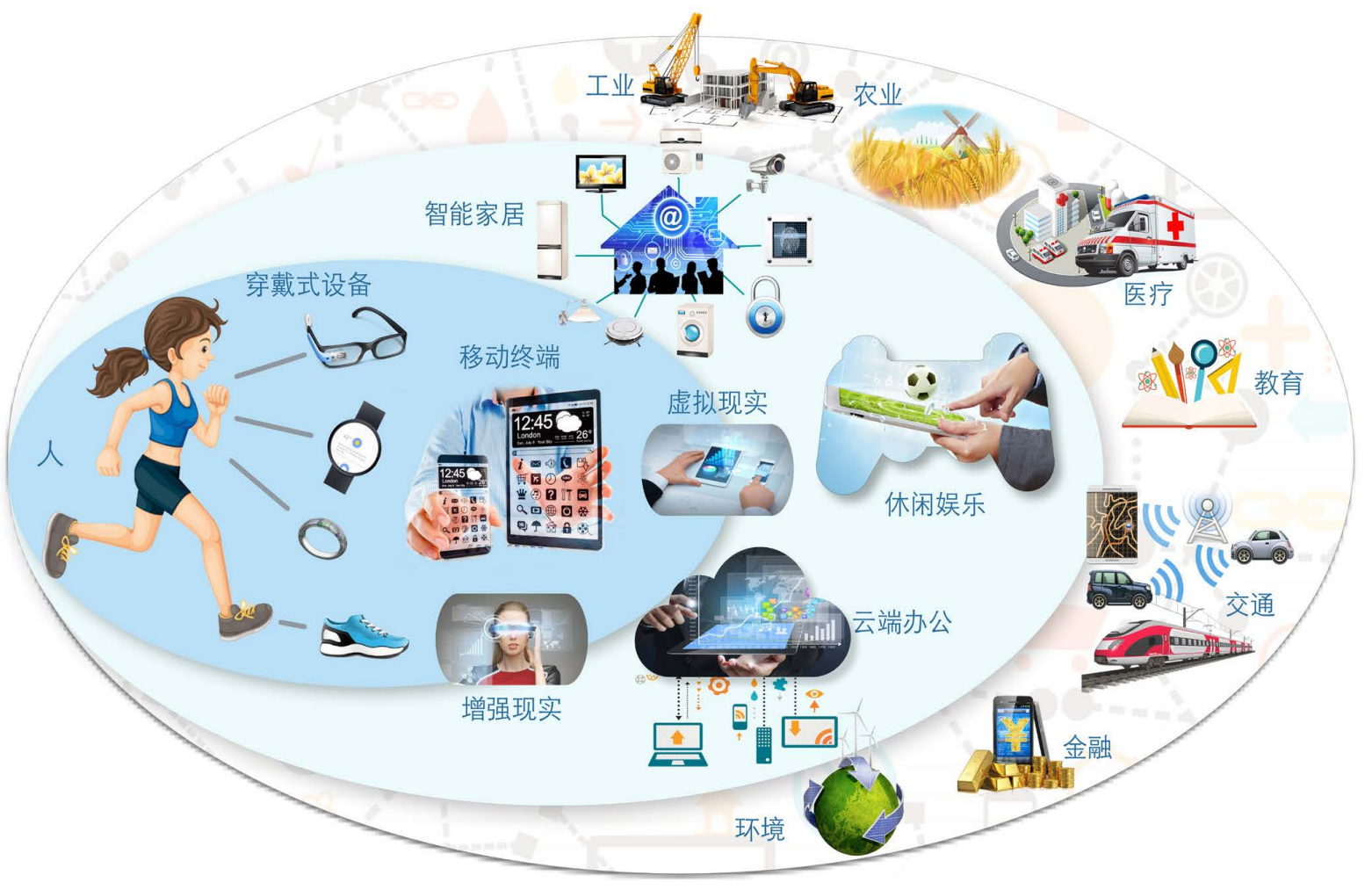

图 $15 \mathrm{G}$ 业务总体愿景 ${ }^{[1]}$

然而, 面对移动互联网和物联网带来的巨大的业务需求, 传统移动通信频谱资源已趋饱 和, 因此毫米波频段正成为产业界和学术界广泛研究的课题。目前, 通信产业界也达成共识: 未来 $5 \mathrm{G}$ 网络将采用低频段（<6 $<\mathrm{GHz}$ ) 蜂窝进行广域覆盖外加高频段（>6GHz）毫米波进行热 点区域接入相结合的方式: 一部分是基于现有技术的演进, 可以兼容现有的移动通信系统, 频率主要在 $6 \mathrm{GHz}$ 以下; 另一部分则采用 $6 \mathrm{GHz}$ 以上的毫米波频段, 利用毫米波的超高传输速 率实现超密集场景、超高速率要求的热点区域覆盖。目前产业界研究的5G典型候选频段主要 集中在 $15 \mathrm{GHz} 、 28 \mathrm{GHz} 、 38 \mathrm{GHz}$ 和 $72 \mathrm{GHz}^{[4-5]}$ 。

\section{2. 产业界主要公司毫米波5G研究进展}

本部分将选取中欧美日韩五大 $5 \mathrm{G}$ 推动国最具代表性的五家产业界公司（欧盟爱立信、中 国华为、韩国三星、美国高通、日本NTT DoCoMo），探讨毫米波5G领域的最新研究进展 ${ }^{[4-7]}$ 。

\section{1 爱立信研究进展}

2014年，爱立信发布了的全球首台5G移动终端原型。2016年2月，在巴塞罗那世界移动 通信大会上, 爱立信采用 $15 \mathrm{GHz}$ 频段实现了 $20-25 \mathrm{Gbps}$ 的峰值速率。2016年11月, 爱立信、SK 电讯和宝马联合在韩国完成了 $28 \mathrm{GHz}$ 毫米波5G户外车辆移动测试。2017年4月, 爱立信和俄 罗斯最大电信运营商MTS在莫斯科开展了 $5 \mathrm{G}$ 技术测试: 此次测试采用了Multi-User、Massive MIMO、光速追踪等技术, 基站使用14.5-15.3GHz的频段, 以智能手机为载体, 实现了 $25 \mathrm{Gbps}$ 的数据传输速度。2018年1月, 爱立信发布了首个 $5 \mathrm{G}$ 小基站 “ $5 \mathrm{G}$ Radio Dot”, 用于满足5G时 代室内移动宽带的性能要求。2018年4月, 爱立信联合其他 25 家创始成员, 发起了 “工业互联 与自动化 $5 \mathrm{G}$ 联盟”。

\section{2 华为研究进展}

2016年2月巴塞罗那移动世界大会上, 华为联手德国电信现场演示了毫米波 $5 \mathrm{G}$ 多用户 MIMO技术, 在73GHz毫米波段上达到了70Gbps的超高传输速率。2016年7月, 华为联手沃达 
丰在英国完成了 $5 \mathrm{G}$ 毫米波（E-Band, $71-76 \mathrm{GHz}$ ）现场测试：利用高强度物理反射的单用户 MIMO实现了单用户设备达到20Gbps的峰值传输速度, 并通过多用户MIMO实现远距单用户 设备达到10Gbps的峰值传输速度。2016年11月，华为联手澳大利亚运营商Optus合作完成了毫 米波 $5 \mathrm{G}$ 网络测试, 使用 $73 \mathrm{GHz}$ 频段实现了 $35 \mathrm{Gbps}$ 的传输速率。2016年11月 17 日, 在 $3 \mathrm{GPP}$ RAN1 87 次会议的 $5 \mathrm{G}$ 短码方案讨论中, 华为的Polar Code (极化码) 方案, 最终战胜列强, 成为 $5 \mathrm{G}$ 增强移动宽带场景控制信道编码最终方案。2017年6月, 华为率先完成了 IMT-2020(5G) 推进组我国5G第二阶段测试。2018年2月, 在2018世界移动通信大会前夕, 华为正式面向全 球发布了首款符合 $3 \mathrm{GPP}$ 标准的5G商用芯片巴龙5G01 (Balong 5G01), 和基于该芯片的首款 $3 \mathrm{GPP}$ 标准 $5 \mathrm{G}$ 商用终端华为 $5 \mathrm{G} \mathrm{CPE}$ 。

\section{3 三星研究进展}

2013年5月, 三星首次完成了 $28 \mathrm{GHz}$ 频段 $5 \mathrm{G}$ 测试, 数据传输速率达到1Gbps。2015年9月, SK电信联合三星在韩国盆塘部署了一套毫米波5G通信系统。2016年9月，三星美国子公司联 合美国电信运营商T-Mobile, 在室外环境进行了 $28 \mathrm{GHz}$ 毫米波频段5G初步测试。2017年5月, 三星电子美国公司宣布联手思科及Verizon, 成功进行了业界首次多供应商端到端5G网络现场 测试。2018年2月平昌冬奥会上，三星、韩国电信等公司率先为冬奥会用户提供了5G应用服 务, 这也是 $5 \mathrm{G}$ 的全球首秀; 三星在冬奥会的多个场馆提供了 200 台支持 $5 \mathrm{G}$ 的平板设备, 方便 用户使用 $5 \mathrm{G}$ 专用设备查看比赛内容和赛程数据。

\section{4 高通研究进展}

2016年2月世界移动通信大会上，高通展示了28GHz移动化毫米波原型机。2016年10月， 高通发布首款 $5 \mathrm{G}$ 调制解调器骁龙X50: 该调制解调器支持 $28 \mathrm{GHz}$ 频段毫米波频段, 采用支持 自适应波束成形和波束追踪的MIMO技术，可支持高达每秒5Gbps的峰值下载速度。2017年2 月, 高通联手中兴通讯和中国移动, 开展了基于5G新空口规范的互操作性测试和OTA外场试 验。2017年10月, 高通展示了全球第一款5G手机样机。2018年3月, 高通骁龙855曝光, 高通 将这一代顶级SoC称为 “骁龙 855 Fusion”: 直接搭载 $5 \mathrm{G}$ 基带骁龙X50, 采用 $28 \mathrm{~nm}$ 工艺, 数据 传输速度达到 $5 \mathrm{Gbps}$, 支持 $3.5 \mathrm{GHz} / 4.5 \mathrm{GHz}$ 中频 ( Sub $6 \mathrm{GHz}$ ), 也支持 $28 \mathrm{GHz} / 38 \mathrm{GHz}$ 的高频 (毫 米波）。

\section{5 日本NTT DoCoMo研究进展}

该公司认为未来 $5 \mathrm{G}$ 无线接入是由为高频段无线接入技术和LTE-A技术的结合。5G网络应 该由覆盖层和容量层两部分组成。容量层: 高频段满足高速数据需求, 覆盖层: 低频率完成 移动覆盖。2015年10月, 诺基亚与NTT DoCoMo在东京新城森大厦内的商业设施, 合作实施 了使用毫米波的 $5 \mathrm{G}$ 验证实验: 中心频率为 $73.5 \mathrm{GHz}$, 系统带宽为 $1 \mathrm{GHz}$, 最大吞吐量超过每秒 2Gbit。2016年11月, DoCoMo联合三星完成了高速列车5G测试, 使用28GHz高频频带, 在时 速高达 150 公里车辆的移动设备上实现了超过 $2.5 \mathrm{Gbps}$ 的数据速率。2017年6月, 在 2017 年第三 届东京湾全球 $5 \mathrm{G}$ 峰会期间, NTT DOCOMO联合华为首次完成基于 $3 \mathrm{GPP} 5 \mathrm{G}$ 新空口的 $39 \mathrm{GHz}$ 高频技术测试, 实现了三方实时4K高清视频会议。2018年1月, NTT DoCoMo公司与诺基亚 首次正式签署5G移动网络设备协议, NTT将在 2020 年前启动商业化 $5 \mathrm{G}$ 网络服务, 及时赶上东 京奥运会。

\section{3. 结束语}

本文选取了中欧美日韩五大5G推动国最具代表性的五家产业界公司（欧盟爱立信、中国 华为、韩国三星、美国高通、日本NTT DoCoMo），探讨了毫米波5G领域的最新研究进展。 通过分析, 我们发现未来 $5 \mathrm{G}$ 网络将采用低频段 ( $<6 \mathrm{GHz})$ 蜂窝进行广域覆盖外加高频段 ( $>6 \mathrm{GHz}$ ) 毫米波进行热点区域接入相结合的方式: 一部分是基于现有技术的演进, 可以兼 
容现有的移动通信系统, 频率主要在 $6 \mathrm{GHz}$ 以下; 另一部分则采用 $6 \mathrm{GHz}$ 以上的毫米波频段, 利用毫米波的超高传输速率实现超密集场景、超高速率要求的热点区域覆盖。

\section{致谢}

本文由深圳市个人创客项目（GRCK2017042415235934），深圳市基础研究项目 （ JCYJ20170817113758285 、 JCYJ20170307164201104 、 JCYJ20160329161334453 、 JCYJ20170817112527562 、 JCYJ20170412153845293 、 JCYJ20170307164247428 、 JCYJ20170817113844300 、 JCYJ20170817112708243 、 JCYJ20170817113029596 、 JCYJ20170817112848591、JCYJ20170412153812353、JCYJ20170307172513653），中国博 士后基金面上资助项目（2017M620523、2017M622619），深圳市技术攻关项目 (JSGG20170414140411874), 国家自然科学基金项目（61574005）, 深港产学研基地产学 研项目（场效应太赫兹器件的关键技术研究）资助。

\section{References}

[1] China Academy of Information and Communications Technology, "The general development trend of 5G", 2015.12.

[2] Guoqing Hu, Anpeng Huang, Tammy Chang, Xiang Cheng, Hequan Wu, LinzhenXie, Anshi $\mathrm{Xu}$, Zhangyuan Chen, "A Sensor-Based Seamless Handover Solution for Express Train Access Networks (ETANs),” IEEE Communications Letters, vol.16, no.4, pp. 470-472, April 2012.

[3] Guoqing Hu, Anpeng Huang, Ruisi He, Bo Ai, Zhangyuan Chen, "Theory analysis of the handover challenge in Express Train Access Networks (ETAN)", China Communications, vol. 11, no.7, pp. 92-98, July 2014.

[4] A. Gupta, R. K. Jha, "A Survey of 5G Network: Architecture and Emerging Technologies", IEEE Access, vol.3, pp.1206-1232, 2015.08.

[5] Jeffrey G. et.al, "What Will 5G Be?", IEEE Journal on Selected Areas in Communications, vol.32, no.6, pp.1065-1082, 2014.07.

[6] T. S. Rappaport, Y. Xing, G. R. MacCartney, Jr., A. F. Molisch, E. Mellios, and J. Zhang, "Overview of millimeter wave communications for fifth-generation $(5 \mathrm{G})$ wireless networks," IEEE Transactions on Antennas and Propagation, vol.65, no.12, pp.6213-3230, Dec. 2017.

[7] J. Huang, C.-X. Wang*, R. Feng, J. Sun, W. Zhang, and Y. Yang, "Multi-frequency MmWave massive MIMO channel measurements and characterization for $5 \mathrm{G}$ wireless communication systems,” IEEE J. Sel. Areas Commun., vol. 35, no. 7, pp. 1591-1605, July 2017. 